\title{
Double Slit Experiment with Microorganisms
}

Author: John Taylor

Recently there was an article published on OSF preprints ${ }^{1}$, titled 'Additional information and clarifications surrounding the Many Dimensions interpretation of Quantum Mechanics and a new thought experiment', which presents a new variation of the double slit experiment involving 'flies'.

The experiment imagines flies being placed in a 'box' one at a time, with a double slit set up in the middle. The flies then make the conscious decision to travel through one of the slits, from one end of the box, to a detector screen at the opposite end, which contains food. Although this is an interesting idea, it would be better to use single celled microorganisms, so that an interference pattern is detectable. Moreover the width of the slit would have to be roughly the same size as the object's de broglie wavelength in order for a diffraction pattern to be exhibited(which is much more feasible with microorganisms compared to flies). However it is still difficult to create a slit the size of their wavelength because their de broglie wavelength is smaller than themselves. But it could be possible to achieve some form of diffraction even if the slits may not be the same size as the microorganisms the object's wavelength, because they are significantly smaller compared to flies(especially if many microorganisms are used at once because this would maximise interference). Another reason why it is beneficial to use single celled microorganisms (instead of flies) is because they are capable of making the types of decisions in order for this experiment to work, which is that they have the ability to travel towards food. In fact experiments have proven that single celled organisms such as E. coli can sense molecule gradients as small as one molecular micron of 1000 molecules per cell volume ${ }^{2}$. Furthermore it is also best to use

\footnotetext{
${ }^{1}$ Taylor, John j. 2018. "Additional Information and Clarifications Surrounding the Many Dimensions Interpretation of Quantum Mechanics, and a New Thought Experiment." Open Science Framework. April 23. doi:10.31219/osf.io/xzgw6.

${ }^{2}$ Roberts, Mark A.J.,Antonis Papachristodoulou, and judith P. Armitage. "Adaptation and Control Circuits in Bacterial Chemotaxis."Biochemical Society Transaction 38,no. 5(2010): 1265-269.doi:10.1042/bst0381265.
} 
unicellular microorganisms because the de Broglie wavelength is far too small for interference to be noticeable when using flies. For example a fly with a mass of $2 \mathrm{e}-07 \mathrm{~kg}$ and traveling at a velocity of $22.2 \mathrm{~m} / \mathrm{s}$, has a de broglie wavelength of $1.49 \mathrm{e}-28 \mathrm{~m}$. However, taking a microbe with an estimated mass of $2 \mathrm{e}-17 \mathrm{~kg}$ and traveling at a velocity of $22.2 \mathrm{~m} / \mathrm{s}$, has a much larger wavelength of $1.49 \mathrm{e}-18 \mathrm{~m}$. Additionally it would be best to use airborne bacteria, for such an experiment, an example being Pertussis or Diphtheria. The article also says that this experiment would be able to determine whether conscious beings collapse the wave function. Furthermore philosophical conclusions are reached, one of which is that we are no different to other objects set in motion (e.g. a tennis ball) if an interference pattern is produced. This is because the decisions made by the microorganisms to travel through one of the slits(to the food), would behave in the same probabilistic manor as a series of electrons being fired though the double slit(if an interference pattern is gets produced), which demonstrates that conscious decisions exhibit the same properties as objects which are not alive, which ultimately implies that we are no different to all other objects set in motion. However there are scientific conclusions, which could be drawn up from this experiment. One of which is that rather than telling us about our status in the universe, it tells us whether the decisions we make are quantum mechanical or not, depending on whether an interference pattern is produced, since the microorganism would make the decision to travel through the double slit to reach the food on the other side. It is also worth mentioning that a double slit experiment could also be performed with microorganisms but without them making the decision to travel through the slits. Instead the microorganisms could be fired through the slits in the same way as electrons are fired in the original double slit experiment. An experiment like this would be an excellent proof of quantum superposition at the macroscopic level. In addition to this it would be a whole new variation of the double slit experiment and would demonstrate the manifestation of quantum superposition in living organisms. In order to minimise the effect of decoherence during such an experiment, a low pressure environment should be used, possibly even a vacuum. Spore forming bacteria could be used because research on the international space station has proved that they are capable of surviving the vacuum of outer space, and because of the relatively small size ${ }^{3}$. Moreover spore forming bacteria cannot

\footnotetext{
${ }^{3}$ Horneck, G., Moeller, R., Cadet, J., Douki, T., Mancinelli, R., Nicholson, W., Panitz, C., Rabbow, E., Rettberg, P., Spry, A., Stackebrandt, E., Vaishampayan, P. and Venkateswaran, K. (2012). Resistance of Bacterial Endospores to Outer Space for Planetary Protection
} 
be used in the version of this experiment involving decision making because they are not capable of making decisions, so a method needs to be developed which involves the microorganisms being able to make the decision to travel through the double slit(by not being in a vacuum, but an environment which they can live in), and also not decohering at the same time. Furthermore a method would have to be developed so that these organisms are fired at high speed in a coherent beam in order to maximise their chance of creating an interference pattern.

\section{Bibliography:}

1. Taylor, John j. 2018. "Additional Information and Clarifications Surrounding the Many Dimensions Interpretation of Quantum Mechanics, and a New Thought Experiment." Open Science Framework. April 23. doi:10.31219/osf.io/xzgw6.

2. Roberts, Mark A.J.,Antonis Papachristodoulou, and judith P. Armitage. "Adaptation and Control Circuits in Bacterial Chemotaxis."Biochemical Society Transaction 38,no. 5(2010): 1265-269.doi:10.1042/bst0381265.

3. Horneck, G., Moeller, R., Cadet, J., Douki, T., Mancinelli, R., Nicholson, W., Panitz, C., Rabbow, E., Rettberg, P., Spry, A., Stackebrandt, E., Vaishampayan, P. and Venkateswaran, K. (2012). Resistance of Bacterial Endospores to Outer Space for Planetary Protection Purposes-Experiment PROTECT of the EXPOSE-E Mission. Astrobiology, [online] 12(5), pp.445-456. Available at: https://www.ncbi.nlm.nih.gov/pmc/articles/PMC3371261/ [Accessed 12 Oct. 2018].

Purposes-Experiment PROTECT of the EXPOSE-E Mission. Astrobiology, [online] 12(5), pp.445-456. Available at: https://www.ncbi.nlm.nih.gov/pmc/articles/PMC3371261/ [Accessed 12 Oct. 2018]. 